\title{
Nuclear Expression of CD133 Is Associated with Good Prognosis in Patients with Colorectal Adenocarcinoma
}

\author{
YONG-MOON LEE ${ }^{1}$, MIN-KYUNG YEO ${ }^{2}$, IN-OCK SEONG ${ }^{2}$ and KYUNG-HEE KIM ${ }^{2}$ \\ ${ }^{I}$ Department of Pathology, Dankook University, School of Medicine, Cheonan, Republic of Korea; \\ ${ }^{2}$ Department of Pathology, Chungnam National University School of Medicine, Daejeon, Republic of Korea
}

\begin{abstract}
Background/Aim: Prominin-1 (CD133) has been suggested as a potential marker of cancer stem cells (CSCs) in various cancer types. The aim of this study was to compare CD133 expression between adenoma cells, colorectal adenocarcinoma (CRAC) cells, and tumor microenvironment cells (TMEs) in terms of the adenoma-carcinoma sequence and to investigate the clinicopathological value of CD133 expression in CRACs as a prognostic marker. Materials and Methods: A total of 58 adenomas and 132 primary and 27 metastatic CRACs were examined for CD133 expression by immunohistochemistry. The cytoplasmic and nuclear expression levels of CD133 were scored by semi-quantitative methods. Results: Adenomas showed significantly lower cytoplasmic and nuclear CD133 expression than CRACs $(p<0.001)$. Among the CRACs, primary CRACs demonstrated higher cytoplasmic and nuclear expression levels of CD133 than metastatic CRACs $(p<0.001)$. Furthermore, decreased nuclear CD133 expression in CRACs was correlated with a poor outcome, including disease-free survival (DFS), by univariate and multivariate analyses ( $p=0.012$ and $p=0.039$ ). For TMEs, adenomas showed a significantly higher CD133 expression than CRACs $(p<0.001)$, and decreased expression of CD133 in CRACs was correlated with shorter DFS by univariate and multivariate analyses ( $p=0.004$ and $p=0.042$ ). Conclusion: Cytoplasmic and nuclear CD133 expression in CRAC cells and TMEs may play an important role in early CRAC carcinogenesis, while decreased CD133 nuclear expression in CRAC cells may contribute to CRAC metastasis. Further prognostic and therapeutic stratification may be performed according to CD133 localization.
\end{abstract}

Correspondence to: Kyung-Hee Kim, Department of Pathology, Cancer Research Institute, Chungnam National University, School of Medicine, 266 Munhwa Street, Jung-gu, Daejeon 35015, Republic of Korea. Tel: +82 425808238, Fax: +82 422807189, e-mail: phone330@cnu.ac.kr

Key Words: CD133, colorectal adenocarcinoma, adenoma, stem cell, tumor microenvironment.
Colorectal adenocarcinoma (CRAC) is one of the most common types of lethal cancer in human. Its treatment is determined primarily by the TNM staging system; however, technical developments in molecular characterization have enabled a better understanding of not only the etiology, but also the response to stage-specific treatment of CRAC. Cancer stem cells (CSCs) or tumor-initiating cells are thought to be responsible for the recurrence or distant metastasis of CRAC, as well as for chemo- and radioresistance $(1,2)$. The isolation and characterization of CSCs are of great importance in the development of new therapeutic strategies targeting these cells. The CSC theory was initially proposed in leukemia and subsequently in solid tumors, and many studies have focused on prominin-1 (CD133) as a candidate-specific marker for CSCs (3).

$\mathrm{CD} 133$ is a $120-\mathrm{kDa}$ transmembrane glycoprotein, preferentially localized in membrane protrusions and microvilli, and may play a role in cell polarity and migration via cell-cell and cell-matrix interactions. In addition, several studies have demonstrated that CD133 expression may serve as an independent marker of prognosis and chemoresistance in CRAC $(4,5)$. However, other studies have failed to establish a correlation between CD133 expression and tumor progression or survival, thus questioning its predictive value in CRAC (3). CD133 expression is observed in normal colon mucosa and has been also found in other normal tissues. CD133-positive cells are distributed uniformly along the epithelium lining without increased expression at the base of colonic crypts (6). Cytoplasmic expression is thought to occur when CD133 is transported from the plasma membrane to the cytoplasm during endocytosis (7), which is putative in CRAC (3).

Considering that tumor microenvironment cells (TMEs) consist of tumor-infiltrating inflammatory cells, extracellular matrix, blood vessels, and a variety of associated stromal cells and soluble proteins (8) that have been known to shape tumor evolution $(9,10)$, it could be speculated that CD133positive TMEs may contribute to tumor development and its progression.

To evaluate the role of CD133 in early carcinogenesis in the colorectum, we compared the degree of cytoplasmic and 
nuclear CD133 expression between adenomas and primary and metastatic CRACs. The distribution of CD133 in tumor cells and TMEs was investigated using tissue microarrays (TMAs) of human CRACs, applying a semi-quantitative method to determine the correlation between CD133 expression and clinicopathological parameters including patient outcome.

\section{Materials and Methods}

Patients and specimens. Formalin-fixed paraffin-embedded (FFPE) tumor samples from 58 patients with low-grade adenomas, 132 patients with primary CRAC and 27 patients with metastatic CRAC were collected from the Department of Pathology of Chungnam National University Hospital from 2002 to 2015. The specimens were provided by the National Biobank of Korea, Chungnam National University Hospital, Daejeon, South Korea. The immunohistochemical studies using FFPE tissue waived the prerequisite for informed consent for a retrospective comparison study using FFPE tissues. The local human research ethics committee of Chungnam National University Hospital and the Institutional Review Board of Chungnam National University Hospital (CNUH 2016-04-038) approved this study.

Two independent pathologists (Y-ML and K-HK) re-evaluated all samples for grade and histological type. Additional clinical data including sex, age, tumor size, histologic grade, pathologic TNM stage, and disease-free and overall survival rates were also collected. No patients had received chemotherapy or radiation before the operation. Tumor staging was performed in accordance with the guidelines of the 8th edition of TNM Staging in Colorectal Cancer (11).

TMA construction and immunohistochemistry (IHC) analysis. A TMA system was used to produce 2-mm-thick paraffin-embedded TMA sections containing 30 holes for tissue columns. Core tissue biopsy samples were taken from individual paraffin-embedded sections and arranged in new recipient paraffin blocks. The TMA blocks were cut into $4-\mu \mathrm{m}$ sections and placed on super frostcharged glass microscope slides. The embedded tissue sections on the microslides were deparaffinized with xylene and hydrated in a graded alcohol series. The sections were heated in a pressure cooker containing $10 \mathrm{mmol} / \mathrm{l}$ sodium citrate $(\mathrm{pH} 6.0)$ for $3 \mathrm{~min}$ at full power for antigen retrieval. Blockade of endogenous peroxide activity was performed using $0.03 \%$ hydrogen peroxide containing sodium azide for $5 \mathrm{~min}$. The sections were incubated at room temperature for $1 \mathrm{~h}$ with a primary monoclonal mouse antibody to CD133 (diluted 1:100; Novus Biologicals, Littleton, CO, USA). The samples were washed and then incubated in labelled polymer-HRP anti-mouse immunoglobulins (Dako EnVision+system-HRP [DAB], Dako, Carpinteria, CA, USA) for an additional $20 \mathrm{~min}$ at room temperature, followed by additional washing. After rinsing, the chromogen was developed for $2 \mathrm{~min}$. The slides were then counterstained with Mayer's hematoxylin, dehydrated, and cover slipped. The primary antibody was omitted in the negative controls. All immunostained slides were digitally scanned using a scanscope (Aperio ScanScope CS System, Vista, CA, USA). Blind CD133 immunostaining evaluation and independent observations were simultaneously performed. The modified Allred scoring system was used for evaluating both the intensity of the immunohistochemical staining and the proportion of stained CRAC cells (12). Staining intensity was scored as 0 (negative), 1 (weakly positive), 2 (moderately positive), or 3 (strongly positive). The percentage of CD133-positive cells was also scored into five categories, with a score of 1 for $<1 \%, 2$ for $<10 \%, 3$ for $<33.3 \%, 4$ for $<66.6 \%$, and 5 for $66.6-100 \%$ positivity. The intensity score was multiplied by the percentage score to quantify CD133 expression, for which cytoplasmic and nuclear expression were evaluated separately. For categorical survival analyses, expression at median values was regarded as high CD133 expression, with staining scores defined as follows: for cytoplasmic expression, 0-9 was considered low expression, while scores above 9 were considered high expression. For nuclear expression, scores of 0-6 and above 6 were considered low and high expression, respectively.

When assessing CD133 expression in TMEs, the tumor proportion scoring system (TPS) was applied according to intensity and localization (cytoplasmic or nuclear expression) in TMEs covering the tumor area occupied by tumor cells, associated with intratumoral and contiguous peritumoral stroma. A modified VENTANA PD-L1 (SP142) assay was used $(13,14)$. The TPS was classified into four categories, with a score of 0 for 0,1 for $<1 \%, 2$ for $\geq 1 \%$ and $<5 \%, 3$ for $\geq 5 \%$ and $<10 \%$, and 4 for $\geq 10 \%$.

Statistical analysis. The association between cytoplasmic and nuclear expression of CD133 in adenomas/CRACs and categorical variables were analyzed by the Mann-Whitney $U$-test and Kruskal-Wallis test, as appropriate. Postoperative overall survival (OS) and disease-free survival (DFS) curves were estimated using the Kaplan-Meier method, and differences in the survival distributions were evaluated using log-rank tests. Univariate and multivariate survival analyses were performed using Cox's proportional hazard regression model. Statistical significance was set at $p<0.05$ (IBM SPSS Statistics for Windows, version 24.0, IBM Corp., NY, USA and R version 3.4.3).

\section{Results}

CD133 expression in the non-neoplastic mucosa of the colorectum was exclusively localized to the luminal side of the cell membrane. CD133 was not expressed in the cytoplasm but was detected on the luminal side of the cytoplasmic membrane in the non-neoplastic mucosa. The cytoplasmic and nuclear expression levels of CD133 in adenomas were significantly lower than those in CRACs $(p<0.001)$ (Figures 1 and 2).

The cytoplasmic and nuclear expression levels of CD133 were measured in 132 primary and 27 metastatic CRAC samples, as summarized in Table I. In metastatic CRAC, nuclear CD133 expression was decreased in samples with a poor or undifferentiated histologic grade compared to those with a well or moderate histologic grade $(p=0.049)$. Higher CD133 expression in TMEs showed positive correlations with a lower histologic grade (well or moderate) and early pathologic stage (Stage I) in the primary CRAC group $(p<0.006$ and $p=0.020)$.

DFS and OS analyses were performed on a total of 132 primary and 27 metastatic CRACs. Low nuclear CD133 expression in CRAC cells and decreased CD133 expression 
A

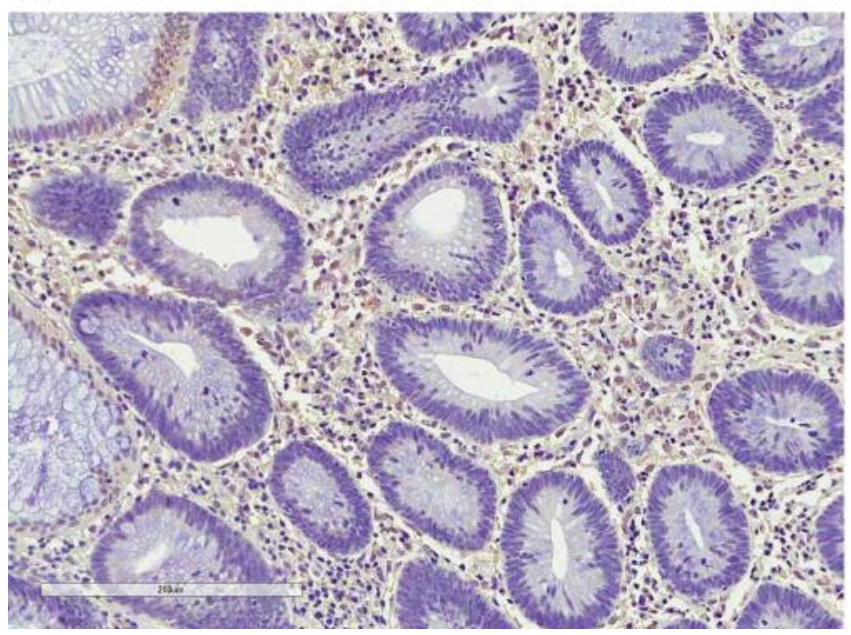

B

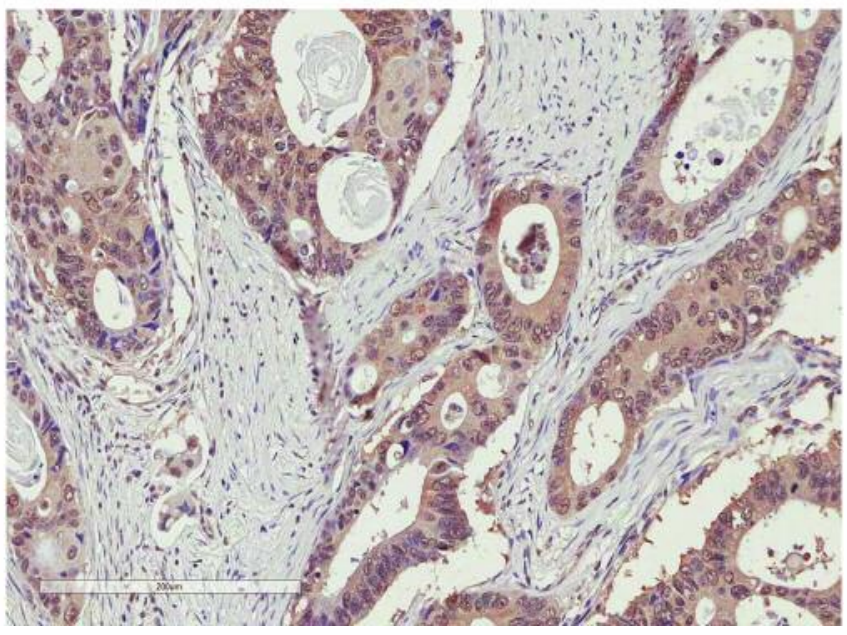

Figure 1. CD133 expression in adenoma and CRAC patients. (A) The very low cytoplasmic and nuclear CD133 expression of tumor cells is shown in contrast to the high CD133 expression in TME cells in adenomas (scale bar $=200 \mu \mathrm{m})$. (B) The high nuclear CD133 expression of invasive tumor cells is shown in comparison to the low expression in TME cells, which is in contrast to that in adenomas (scale bar=200 $\mu \mathrm{m}$ ).

in TMEs were statistically correlated with shorter DFS in CRAC patients $(p=0.012$ and $p=0.004)$ (Figure 3). Univariate and multivariate analyses using Cox's proportional hazard regression model were performed for sex, age, histologic grade, pathologic TNM stage, CD133 expression in CRAC cells and the degree of CD133 expression in TME cells (Tables II and III). The multivariate analysis of the Cox model showed lower nuclear expression of CD133 in CRAC cells and decreased CD133 expression in TMEs, suggesting that CD133 served as a favorable prognostic factor indicative of shorter DFS ( $p=0.039$ and $p=0.042$ ) (Table III).

\section{Discussion}

CD133 is a cell surface transmembrane glycoprotein within the cholesterol-rich lipid raft that was initially identified in the neuroepithelium of endothelial progenitor cells. Although its cellular function is not clear (7), CD133 is an important marker for a number of different CSC lineages. CD133 has been proposed as a specific marker for the isolation of CSCs from brain tumors and prostate and liver carcinoma tissue and has also been suggested as a candidate marker for CSCs in CRACs (3).

Recent studies have shown two distinct staining patterns for CD133: apical/endoluminal membrane staining and cytoplasmic staining (7). In fact, the normal colon mucosa includes CD133-positive cells (mainly goblet cells) at all levels of the crypts. Examination of whole tumors revealed weak CD133 expression in the non-neoplastic colon mucosa

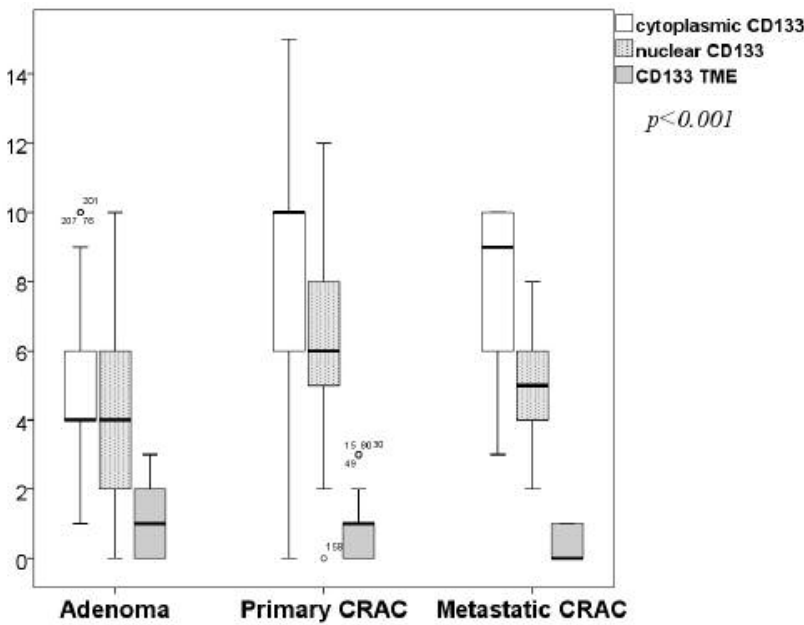

Figure 2. A total of 58 adenoma cases, 132 primary CRAC cases and 27 metastatic CRAC cases were examined. CD133 expression was assessed with a Kruskal-Wallis test ( $p<0.001$ for each). The line in the middle of each box is the median. The box length indicates the interquartile range. The ends of the whiskers represent maximum and minimum values.

compared to that in dysplastic and neoplastic tissues, with most of this expression localized to cells at the base of the crypts. Some authors proposed that apical/endoluminal membranous CD133 staining is a characteristic of welloriented, polarized, and differentiated cells, while cytoplasmic staining may be indicative of putative CSCs (7). In short, the 
Table I. Univariate analysis comparing the nuclear and cytoplasmic CD133 expression levels in tumor cells and CD133 expression in tumor microenvironment cells (TMEs) with clinicopathological variables in CRAC.

\begin{tabular}{|c|c|c|c|c|c|c|c|}
\hline \multirow[t]{2}{*}{ Characteristics } & \multirow[t]{2}{*}{ No. } & \multicolumn{2}{|c|}{ Nuclear CD133 in tumor } & \multicolumn{2}{|c|}{ Cytoplasmic CD133 in tumor } & \multicolumn{2}{|c|}{ CD133 expression in TMEs } \\
\hline & & Mean \pm SD & $p$-Value & Mean \pm SD & $p$-Value & $\operatorname{Mean} \pm \mathrm{SD}$ & $p$-Value \\
\hline \multicolumn{8}{|c|}{ Primary colorectal adenocarcinoma } \\
\hline Age (year) & 132 & & 0.687 & & 0.258 & & 0.672 \\
\hline$<65$ & 77 & $6.5 \pm 2.51$ & & $8.1 \pm 2.65$ & & $0.8 \pm 0.92$ & \\
\hline$\geq 65$ & 55 & $6.3 \pm 2.26$ & & $8.8 \pm 2.76$ & & $0.9 \pm 0.83$ & \\
\hline Gender & 132 & & 0.731 & & 0.071 & & 0.591 \\
\hline Male & 85 & $6.4 \pm 2.33$ & & $8.7 \pm 2.84$ & & $0.8 \pm 0.84$ & \\
\hline Female & 47 & $6.4 \pm 2.55$ & & $7.9 \pm 2.45$ & & $0.9 \pm 0.95$ & \\
\hline Histologic grade & 132 & & 0.624 & & 0.094 & & 0.006 \\
\hline low (I\&II) & 121 & $6.4 \pm 2.47$ & & $8.3 \pm 2.75$ & & $0.9 \pm 0.89$ & \\
\hline High(III\&IV) & 11 & $6.0 \pm 1.55$ & & $9.4 \pm 2.29$ & & $0.2 \pm 0.41$ & \\
\hline Pathologic stage & 132 & & 0.851 & & 0.750 & & 0.020 \\
\hline I & 21 & $6.4 \pm 2.52$ & & $8.4 \pm 2.42$ & & $1.3 \pm 1.11$ & \\
\hline II-IV & 111 & $6.4 \pm 2.39$ & & $8.4 \pm 2.78$ & & $0.75 \pm 0.80$ & \\
\hline Pathologic stage & 132 & & 0.721 & & 0.971 & & 0.105 \\
\hline I and II & 56 & $6.32 \pm 2.61$ & & $8.38 \pm 2.85$ & & $1.00 \pm 0.95$ & \\
\hline III and IV & 76 & $6.46 \pm 2.25$ & & $8.42 \pm 2.63$ & & $0.72 \pm 0.81$ & \\
\hline \multicolumn{8}{|c|}{ Metastatic colorectal adenocarcinoma } \\
\hline Age (year) & 27 & & 0.902 & & 0.309 & & 0.675 \\
\hline$<65$ & 17 & $5.1 \pm 1.78$ & & $7.5 \pm 2.27$ & & $0.3 \pm 0.47$ & \\
\hline$\geq 65$ & 10 & $5.1 \pm 0.45$ & & $8.4 \pm 2.68$ & & $0.4 \pm 0.52$ & \\
\hline Gender & 27 & & 0.451 & & 0.342 & & 0.080 \\
\hline Male & 16 & $5.3 \pm 1.44$ & & $7.3 \pm 2.63$ & & $0.5 \pm 0.52$ & \\
\hline Female & 11 & $4.8 \pm 1.94$ & & $8.6 \pm 1.97$ & & $0.1 \pm 0.30$ & \\
\hline Histologic grade & 27 & & 0.049 & & 0.869 & & 0.576 \\
\hline Low (I\&II) & 23 & $5.3 \pm 1.66$ & & $7.8 \pm 2.49$ & & $0.3 \pm 0.47$ & \\
\hline High(III\&IV) & 4 & $3.8 \pm 0.50$ & & $8.0 \pm 2.31$ & & $0.5 \pm 0.58$ & \\
\hline Pathologic stage & 27 & & N/A & & N/A & & N/A \\
\hline I & 0 & N/A & & N/A & & N/A & \\
\hline II-IV & 27 & $5.1 \pm 1.64$ & & $7.8 \pm 2.42$ & & $0.3 \pm 0.48$ & \\
\hline Pathologic stage & 27 & & 0.519 & & 0.074 & & 0.370 \\
\hline I and II & 1 & $6.0 \pm \mathrm{N} / \mathrm{A}$ & & $3.0 \pm \mathrm{N} / \mathrm{A}$ & & $1.0 \pm \mathrm{N} / \mathrm{A}$ & 1.0 \\
\hline III and IV & 26 & $5.0 \pm 1.66$ & & $8.0 \pm 2.26$ & & $0.3 \pm 0.47$ & \\
\hline
\end{tabular}

N/A: Not applicable; TME: tumor microenvironment cell.

shift of CD133 from a membranous to cytoplasmic localization is thought to underlie the transition of epithelial cells towards a more invasive phenotype (3). Another study on adenomas reported a $17.9 \%$ CD133 positivity rate in colorectal adenomas, a statistically significant increase observed together with the atypical features and size of adenomas (15). Notably, Chen et al. reported that nuclear CD133 conferred optimal clinical outcomes in hepatocellular carcinoma patients regardless of cytoplasmic expression and that cytoplasmic CD133 was related to a poor prognosis (16).

In this study, using a total of 58 adenomas, 132 primary CRAC cases and 27 metastatic CRAC cases, which represented the spectrum from precancerous to cancerous cells known as the adenoma-carcinoma sequence, we compared the degree of cytoplasmic and nuclear CD133 expression between adenomas and CRACs. Our finding that adenomas showed significantly lower cytoplasmic and nuclear CD133 expression than CRACs supports the conclusion that CD133 is increasingly expressed both qualitatively and quantitatively along the path to carcinogenesis. For TMEs, adenomas showed significantly higher CD133 expression than CRACs $(p<0.001)$. We suspect that CD133-positive TMEs in adenomas play a role in tumorigenesis and tumor progression to CRACs. The cytoplasmic and nuclear expression levels of CD133 were the measured in a total of 132 primary and 27 metastatic CRAC samples and evaluated their correlations with clinicopathological parameters to identify prognostic factors. The cytoplasmic and nuclear expression levels of CD133 were decreased in metastatic CRACs and TMEs 
Table II. Univariate analysis of overall survival and disease-free survival in 132 patients with primary CRAC and 27 patients with metastatic CRAC.

\begin{tabular}{|c|c|c|c|c|c|c|}
\hline & \multicolumn{3}{|c|}{ Overall survival } & \multicolumn{3}{|c|}{ Disease-free survival } \\
\hline & $p$-Value & HR & $95 \% \mathrm{CI}$ & $p$-Value & $\mathrm{HR}$ & $95 \% \mathrm{CI}$ \\
\hline Nuclear CD133 & 0.406 & 0.959 & $0.868-1.059$ & 0.012 & 0.855 & $0.756-0.966$ \\
\hline Cytoplasmic CD133 & 0.132 & 1.075 & $0.978-1.181$ & 0.231 & 0.940 & $0.848-1.041$ \\
\hline TME & 0.261 & 0.847 & $0.634-1.131$ & 0.004 & 0.531 & $0.346-0.816$ \\
\hline Gender (male $v s$. female) & 0.343 & 0.785 & $0.476-1.295$ & 0.201 & 1.453 & $0.819-2.578$ \\
\hline Age & 0.011 & 1.030 & $1.007-1.054$ & 0.178 & 0.983 & $0.959-1.008$ \\
\hline Histologic grade $(1 \& 2 v s .3 \& 4)$ & 0.546 & 0.755 & $0.304-1.877$ & 0.535 & 1.342 & $0.530-3.394$ \\
\hline pTNM stage (I\&II vs. III\&IV) & 0.001 & 2.667 & $1.504-4.732$ & $<0.001$ & 5.162 & $2.186-12.188$ \\
\hline
\end{tabular}

HR: Hazard ratio; CI: confidence interval; nuclear CD133: nuclear CD133 expression in cancer cells; cytoplasmic CD133: cytoplasmic CD133 expression in cancer cells; TME: CD133 expression in tumor microenvironment cell.

Table III. Multivariate analysis of overall survival and disease-free survival in 132 patients with primary CRAC and 27 patients with metastatic CRAC.

\begin{tabular}{|c|c|c|c|c|c|c|}
\hline & \multicolumn{3}{|c|}{ Overall survival } & \multicolumn{3}{|c|}{ Disease-free survival } \\
\hline & $p$-Value & HR & $95 \% \mathrm{CI}$ & $p$-Value & HR & $95 \% \mathrm{CI}$ \\
\hline Nuclear CD133 & 0.287 & 0.943 & $0.846-1.051$ & 0.039 & 0.868 & $0.759-0.993$ \\
\hline Cytoplasmic CD133 & 0.205 & 1.067 & $0.965-1.178$ & 0.429 & 0.953 & $0.846-1.073$ \\
\hline TME & 0.416 & 0.877 & $0.639-1.204$ & 0.042 & 0.631 & $0.405-0.984$ \\
\hline Gender (male $v s$. female) & 0.237 & 0.736 & $0.443-1.223$ & 0.573 & 1.186 & $0.655-2.145$ \\
\hline Age & 0.008 & 1.033 & $1.009-1.058$ & 0.685 & 0.994 & $0.968-1.022$ \\
\hline Histologic grade $(1 \& 2 v s .3 \& 4)$ & 0.620 & 0.785 & $0.302-2.041$ & 0.996 & 1.003 & $0.377-2.665$ \\
\hline pTNM stage (I\&II vs. III\&IV) & $<0.000$ & 2.915 & $1.632-5.206$ & 0.001 & 4.538 & $1.885-10.927$ \\
\hline
\end{tabular}

HR: Hazard ratio; CI: confidence interval; nuclear CD133: nuclear CD133 expression in cancer cells; cytoplasmic CD133: cytoplasmic CD133 expression in cancer cells; TME: CD133 expression in tumor microenvironment cell.

compared to primary CRACs $(p<0.001)$. By multivariate analysis, nuclear CD133 expression in CRAC cells and CD133 expression in TMEs was decreased in patients with a poor outcome, including poor DFS $(p=0.039$ and $p=0.042$ ).

This finding suggests that CD133 may play a role in carcinogenesis, especially the early carcinomatous stage, with decreased expression associated with CRAC progression. The different expression of nuclear CD133 between adenomas and primary and metastatic CRAC cells suggests that the "lymphovascular invasion" and "evident recurrence or metastasis" steps may consist of different cancer developmental cells. In fact, Hongo et al. demonstrated that cultured $\mathrm{CD}_{133^{+}}$human colon cells, cloned by a limiting dilution-culture method to produce single-clone $\mathrm{CD} 133^{+}$cells, contained a population of CD133- cells, whereas almost no $\mathrm{CD} 133^{+}$cells were found using flow cytometric analysis among the single-cloned
$\mathrm{CD} 133^{-}$cells after 30 days of culture (6). This result suggests that $\mathrm{CD}_{133^{+}}$cells might be upstream in the differentiation process in relation to $\mathrm{CD} 133^{-}$cells and that $\mathrm{CD}_{133^{+}}$cells can differentiate into $\mathrm{CD} 133^{-}$cells in an irreversible manner. Moreover, the authors reported that $\mathrm{CD}_{133^{-}}$cells showed an increased ability to migrate compared to $\mathrm{CD} 133^{+}$cells and were more resistant to 5fluorouracil than $\mathrm{CD} 133^{+}$cells. These results were supported by another study showing that metastatic colon tumors comprise both $\mathrm{CD}_{133^{+}}$and $\mathrm{CD}^{-} 33^{-}$epithelial cell populations and that both $\mathrm{CD}_{133^{+}}$and $\mathrm{CD} 133^{-}$subsets of $\mathrm{CD}_{133^{+}}$human metastatic colon cancers are capable of initiating tumors in immunodeficient mice with a higher growth rate when the CD133- subset is xenografted (17). Our results showing decreased CD133 expression, prominently nuclear expression, in metastatic CRAC cells compared to primary CRAC cells are consistent with those of the previous study reporting higher cytoplasmic and 

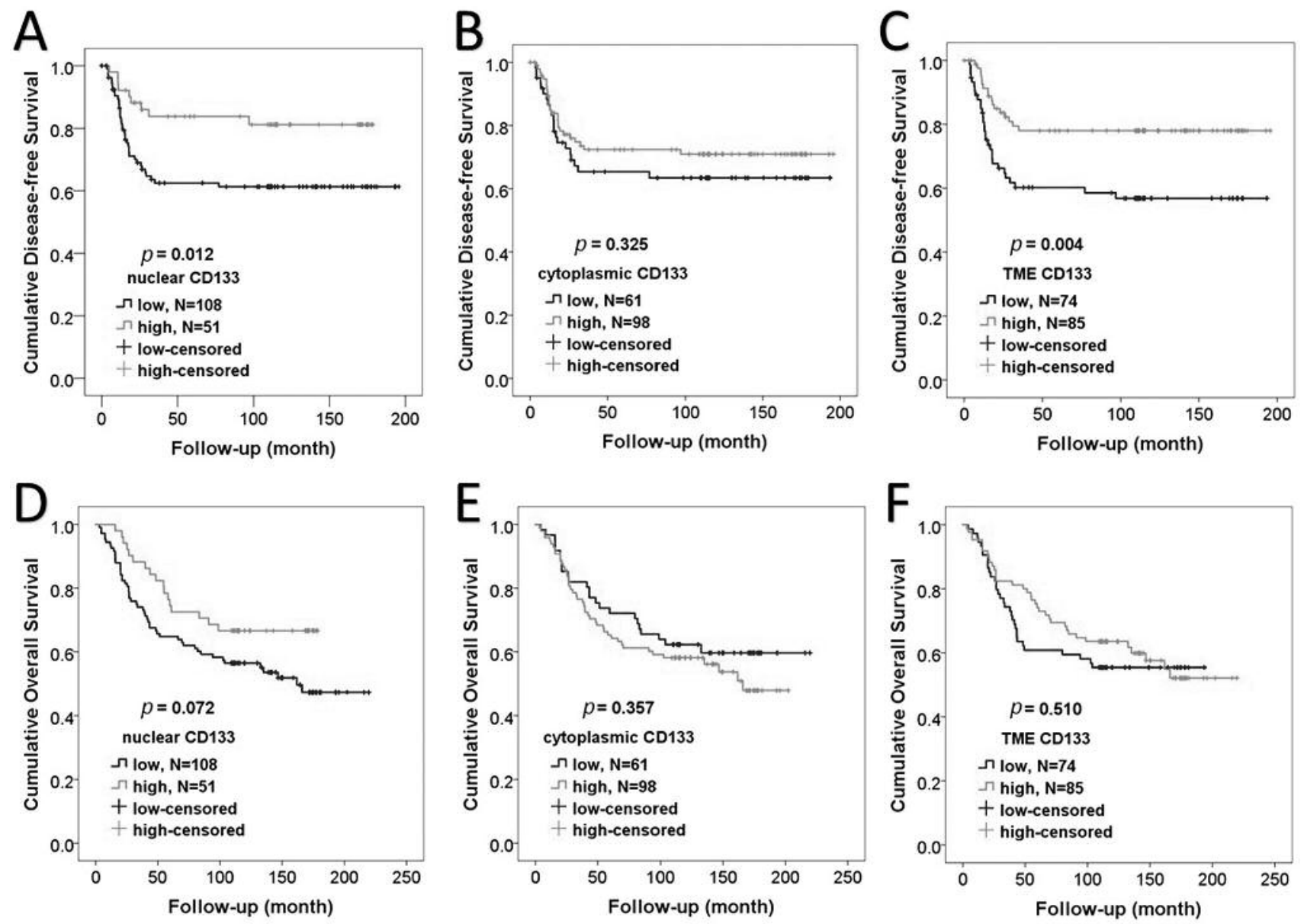

Figure 3. Kaplan-Meier survival curves according to CD133 expression in patients with CRAC ( $n=159)$. (A-C) CD133 expression with diseasefree survival (DFS) in CRAC patients. (D-F) CD133 expression with overall survival (OS) in CRAC patients. The curves show a significant association between decreased nuclear CD133 expression in CRAC cells and a shorter disease-free survival ( $p=0.012)(A)$. The curves show a significant association between decreased CD133 expression in tumor microenvironment cells (TMEs) in CRACs and a shorter disease-free survival $(p=0.004)(C)$.

nuclear expression of CD133 in malignant tumors, whereas nuclear CD133 showed a negative correlation with disease progression in hepatocellular carcinoma (16). Our finding may imply that the change from $\mathrm{CD}_{13}{ }^{+}$cells in primary CRAC to $\mathrm{CD}_{133^{-}}$cells is dominant in metastatic CRAC. A previous study (18) also demonstrated that CRACs showed higher cytoplasmic expression of CD24 than adenomas, another potential marker for tumor stem cells, with an inverse correlation to OS and DFS in CRACs, which are similar observations to those of the present study using CD133 (17, 19-21). In fact, a previous study also showed, using flow cytometric analysis, that the $\mathrm{CD}_{133^{+}}$human colon cancer subset is $\mathrm{CD} 44^{\text {low }} \mathrm{CD} 24^{+}$, whereas the CD133 ${ }^{-}$ subset is $\mathrm{CD} 44^{+} \mathrm{CD} 24^{-}(17)$. As prognostic markers, CD133 and CD24 have shown inconsistencies. However, this may be due to their involvement in unique stages of carcinogenesis and evaluation of their localized expression (cytoplasmic membrane vs. cytoplasm vs. nucleus) in CRACs. More specifically, our results suggest that both cytoplasmic and nuclear CD133 expression play crucial roles in tumorigenesis, with higher expression observed in CRACs than in adenomas. It is likely that $\mathrm{CD} 133^{+}$-initiating carcinogenic cells lose their cytoplasmic CD133 expression but gain nuclear CD133 expression and become more aggressive with tumor progression, suggesting that nuclear CD133 originates from cytoplasmic CD133 and may play a role in the metastatic process. Finally, there seems to be a time-lag in losing CD133 antigenicity throughout the cytoplasm and nucleus; therefore, the prognostic values of cytoplasmic and nuclear CD133 expression are lost after a certain stage of carcinogenesis (22). TMEs are composed of tumor-infiltrating immune cells and non-epithelial cells 
expressing CD133 and should be considered as supporting the establishment of CSCs. There is some evidence that $\mathrm{CD}_{133^{+}}$mesenchymal stem or multipotent stromal cells can contribute to trans-differentiation and tissue repair $(23,24)$. However, based on our investigation, it is unlikely that TMEs also stimulate tumor initiation supported via higher CD133 expression on TMEs in adenomas than in CRACs; in other words, tumor cells are dependent on TMEs only in the initial phase. However, as CRAC progresses, progressive CRAC cells without the support of TMEs are needed for further progression and metastasis. We suggest that CD133positive TMEs and CRAC cells are activated in a specific step during cancer development, where CD133 is expressed in the nuclei of CRAC cells and TMEs and targeting this expression may serve as an effective anti-tumor strategy.

In summary, high cytoplasmic and nuclear expression levels of CD133 in CRACs, compared to adenomas, could indicate early malignant transformation. The observation that the nuclear expression of CD133 in CRAC cells and positivity of CD133 in TMEs were negatively correlated with DFS supports the possibility of a carcinogenesis stepspecific role for CD133 and its potential as a prognostic marker in CRACs, although caution is needed to interpret the localization of CD133, specifically whether there is expression in the nucleus or cytoplasm of tumor cells.

\section{Conflicts of Interest}

All Authors declare that no competing interests exist.

\section{Acknowledgements}

This work was supported by the Basic Science Research Program through the National Research Foundation of Korea (NRF) funded by the Ministry of Education, Science, and Technology (NRF2016R1D1A1B01014311) (KHK).

\section{References}

1 Paldino E, Tesori V, Casalbore P, Gasbarrini A and Puglisi MA: Tumor initiating cells and chemoresistance: which is the best strategy to target colon cancer stem cells? Biomed Res Int 2014: 859871,2014

2 Mathonnet M, Perraud A, Christou N, Akil H, Melin C, Battu S, Jauberteau MO and Denizot Y: Hallmarks in colorectal cancer: angiogenesis and cancer stem-like cells. World J Gastroenterol 20: 4189-4196, 2014

3 Florianova L, Orain M, Têtu B and Doillon C: Histological study of stem-like cells in human colon adenocarcinoma at different stages of the disease. Biotech Histochem 88: 222-234, 2013.

4 Lugli A, Iezzi G, Hostettler I, Muraro MG, Mele V, Tornillo L, Carafa V, Spagnoli G, Terracciano L and Zlobec I: Prognostic impact of the expression of putative cancer stem cell markers CD133, CD166, CD44s, EpCAM, and ALDH1 in colorectal cancer. Br J Cancer 103: 382-390, 2010.
5 Choi D, Lee HW, Hur KY, Kim JJ, Park GS, Jang SH, Song YS, Jang KS and Paik SS: Cancer stem cell markers CD133 and CD24 correlate with invasiveness and differentiation in colorectal adenocarcinoma. World J Gastroenterol 15: 22582264, 2009.

6 Hongo K, Tanaka J, Tsuno NH, Kawai K, Nishikawa T, Shuno Y, Sasaki K, Kaneko M, Hiyoshi M and Sunami E: CD133 (-) cells, derived from a single human colon cancer cell line, are more resistant to 5-fluorouracil (FU) than CD133 (+) cells, dependent on the $\beta 1$-integrin signaling. J Surg Res 175: 278288, 2012.

7 Huang M, Zhu H, Feng J, Ni S and Huang J: High CD133 Expression in the nucleus and cytoplasm predicts poor prognosis in non-small cell lung cancer. Dis Markers 2015: 986095, 2015.

8 Whiteside TL: The tumor microenvironment and its role in promoting tumor growth. Oncogene 27: 5904-5912, 2008.

9 De Wever O and Mareel M: Role of tissue stroma in cancer cell invasion. J Pathol 200: 429-447, 2003.

10 Yuan Y, Jiang YC, Sun CK and Chen QM: Role of the tumor microenvironment in tumor progression and the clinical applications (Review). Oncol Rep 35: 2499-2515, 2016.

11 Amin MB, Edge S, Greene F, Byrd DR, Brookland RK, Washington MK, Gershenwald JE, Compton CC, Hess KR, Sullivan DC, Jessup JM, Brierley JD, Gaspar LE, Schilsky RL, Balch CM, Winchester DP, Asare EA, Madera M, Gress DM and Meyer LR: AJCC cancer staging manual. Eighth edition. Springer International Publishing, Cham, Switzerland, pp. 251274, 2017.

12 Allred DC, Harvey JM, Berardo M and Clark GM: Prognostic and predictive factors in breast cancer by immunohistochemical analysis. Mod Pathol 11: 155-168, 1998.

13 Vennapusa B, Baker B, Kowanetz M, Boone J, Menzl I, Bruey JM, Fine G, Mariathasan S, McCaffery I, Mocci S, Rost S, Smith D, Dennis E, Tang SY, Damadzadeh B, Walker E, Hegde PS, Williams JA, Koeppen H and Boyd Z: Development of a PD-L1 Complementary Diagnostic Immunohistochemistry Assay (SP142) for Atezolizumab. Appl Immunohistochem Mol Morphol, 2018. doi: 10.1097/PAI.0000000000000594. [Epub ahead of print]

14 Sul J, Blumenthal GM, Jiang X, He K, Keegan P and Pazdur R: FDA Approval Summary: Pembrolizumab for the Treatment of Patients With Metastatic Non-Small Cell Lung Cancer Whose Tumors Express Programmed Death-Ligand 1. Oncologist 21: 643-650, 2016.

15 Kazama S, Kishikawa J, Kiyomatsu T, Kawai K, Nozawa H, Ishihara $\mathrm{S}$ and Watanabe T: Expression of the stem cell marker CD133 is related to tumor development in colorectal carcinogenesis. Asian J Surg 41: 274-278, 2017.

16 Chen YL, Lin PY, Ming YZ, Huang WC, Chen RF, Chen PM and Chu PY: The effects of the location of cancer stem cell marker CD133 on the prognosis of hepatocellular carcinoma patients. BMC Cancer 17: 474, 2017.

17 Shmelkov SV, Butler JM, Hooper AT, Hormigo A, Kushner J, Milde T, St Clair R, Baljevic M, White I, Jin DK, Chadburn A, Murphy AJ, Valenzuela DM, Gale NW, Thurston G, Yancopoulos GD, D'Angelica M, Kemeny N, Lyden D and Rafii S: CD133 expression is not restricted to stem cells, and both $\mathrm{CD} 133^{+}$and $\mathrm{CD}^{-} 33^{-}$metastatic colon cancer cells initiate tumors. J Clin Invest 118: 2111-2120, 2008. 
18 Yeo M-K, Lee Y-M, Seong I-O, Choi S-Y, Suh K-S, Song KS, Lee C-S, Kim JM and Kim K-H: Up-regulation of cytoplasmic CD24 expression is associated with malignant transformation but favorable prognosis of colorectal adenocarcinoma. Anticancer Res 36: 6593-6598, 2016.

19 Miki J, Furusato B, Li H, Gu Y, Takahashi H, Egawa S, Sesterhenn IA, McLeod DG, Srivastava S and Rhim JS: Identification of putative stem cell markers, CD133 and CXCR4, in hTERT-immortalized primary nonmalignant and malignant tumor-derived human prostate epithelial cell lines and in prostate cancer specimens. Cancer Res 67: 3153-3161, 2007.

20 Prince ME, Sivanandan R, Kaczorowski A, Wolf GT, Kaplan MJ, Dalerba P, Weissman IL, Clarke MF and Ailles LE: Identification of a subpopulation of cells with cancer stem cell properties in head and neck squamous cell carcinoma. Proc Natl Acad Sci USA 104: 973-978, 2007.

$21 \mathrm{Li} \mathrm{C}$, Lee CJ and Simeone DM: Identification of human pancreatic cancer stem cells. Methods Mol Biol 568: 161-173, 2009.
22 Park EK, Lee JC, Park JW, Bang SY, Yi SA, Kim BK, Park JH, Kwon SH, You JS, Nam SW, Cho EJ and Han JW: Transcriptional repression of cancer stem cell marker CD133 by tumor suppressor p53. Cell Death Dis 6: e1964, 2015.

23 Caplan AI and Dennis JE: Mesenchymal stem cells as trophic mediators. J Cell Biochem 98: 1076-1084, 2006.

24 Phinney DG and Prockop DJ: Concise review: mesenchymal stem/multipotent stromal cells: the state of transdifferentiation and modes of tissue repair--current views. Stem Cells 25: 28962902, 2007

Received June 13, 2018

Revised June 28, 2018

Accepted June 29, 2018 